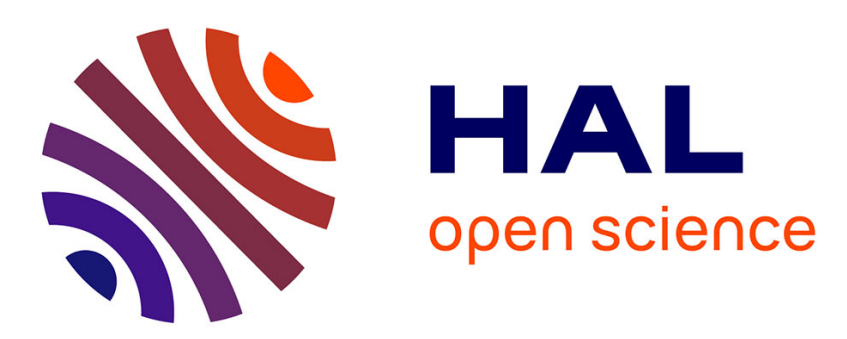

\title{
Optimizing the third harmonic generated from air plasma filaments pumped by femtosecond laser pulses
}

Chenhao Kou, Aurélien Houard, André Mysyrowicz, Y Liu

\section{To cite this version:}

Chenhao Kou, Aurélien Houard, André Mysyrowicz, Y Liu. Optimizing the third harmonic generated from air plasma filaments pumped by femtosecond laser pulses. Journal of the Optical Society of America B, 2019, 36, pp.G13-G18. 10.1364/JOSAB.36.000G13 . hal-02283745

HAL Id: hal-02283745

https://hal-ensta-paris.archives-ouvertes.fr/hal-02283745

Submitted on 11 Sep 2019

HAL is a multi-disciplinary open access archive for the deposit and dissemination of scientific research documents, whether they are published or not. The documents may come from teaching and research institutions in France or abroad, or from public or private research centers.
L'archive ouverte pluridisciplinaire HAL, est destinée au dépôt et à la diffusion de documents scientifiques de niveau recherche, publiés ou non, émanant des établissements d'enseignement et de recherche français ou étrangers, des laboratoires publics ou privés. 


\title{
Optimizing the third harmonic generated from air plasma filaments pumped by femtosecond laser pulses
}

\author{
Chenhao Kou, ${ }^{1}$ Aurélien Houard, ${ }^{2}$ And André Mysyrowicz ${ }^{2, \#}$ \\ YI LIU, ${ }^{1,2, *}$ \\ ${ }^{1}$ Shanghai Key Lab of Modern Optical System, University of Shanghai for Science and Technology, \\ 516, Jungong Road, Shanghai, 200093, China \\ ${ }^{2}$ Laboratoire d'Optique Appliquée, ENSTA ParisTech, CNRS, Ecole polytechnique, 828 Boulevard des \\ Mar'echaux, 91762 Palaiseau, France \\ \#andre.mysyrowicz@ensta-paristech.fr, *yi.liu@usst.edu.cn
}

\begin{abstract}
Third harmonic (TH) generation inside air plasma filaments provides a simple method to convert near-infrared laser pulses in the UV range. Here, we report on the optimization of the third harmonic generation during filamentation in the parameter space of focusing geometry, gas pressure, and incident laser pulse energy. It is observed that, in the nonlinear filamentation regime, the $\mathrm{TH}$ yield is largely dominated by the geometrical focusing condition and gas pressure. Using ambient air as medium for filamentation, an optimal numerical aperture (NA) $=0.07$ was found for laser pulse with peak power close and above the critical power for self-focusing. For more loose focusing geometry (NA < 0.047), reduction of the air pressure can improve the conversion efficiency. In this case, the spatial mode of the third harmonic was also found to be more uniform than in ambient air. With scanning pinhole and crossing-filaments methods, it is revealed that in the situation of optimized focusing geometry $(\mathrm{NA}=0.07)$ the $\mathrm{TH}$ accumulates constructively along the filament and exits at its end. In contrast, for the loose focusing geometry, the destructive interference effect of the TH generated at the leading and trailing edges of the filaments decreases the TH yield efficiency. This study provides a practical guidance for efficient conversion of near-infrared femtosecond pulses to UV domain.
\end{abstract}

(C) 2018 Optical Society of America under the terms of the OSA Open Access Publishing Agreement

\section{Introduction}

An intense laser pulse focused in air generates third harmonic (TH), providing a very simple method for the generation of optical pulses in the ultraviolet range that has been studied since the early days of nonlinear optics [1-4]. With laser peak power higher than the critical power for self-focusing $\mathrm{P}_{\mathrm{cr}}(\sim 5 \mathrm{GW}$ in air), filamentation occurs mainly as a result of the competition of Kerr self-focusing effect and plasma defocusing [5, 6]. In this situation, third harmonic can be generated tens of meters away from the laser system [7], providing an alternative wavelength for remote sensing application based on a single femtosecond laser system. At first sight, third harmonic generation with focused laser pulses in air seems to be simple and well documented $[1-4,8]$. However, in the filamentation propagation regime, several open questions still remain concerning this fundamental process.

First of all, the generation mechanism for the efficient third harmonic from air with focused laser pulses is still not completely clear. It is well known that in the perturbative limit, no net third harmonic is expected to be produced when a laser pulse is focused in an extended media with normal dispersion such as air, even in the case of perfect phasematching [8]. This is due to destructive interferences in the far field between the third harmonics yielded before and after the geometrical focus due to the Gouy phase shift $[2,8]$. To overcome this difficulty, several techniques have been employed, which include a gas cell 
placed before or after the focus [9], a narrow gas jet [10], a non-uniform gaseous medium [11], or employment of wave-guides [12]. With high power laser pulses focused in gases, significant $\mathrm{TH}$ signal has been observed during optical ionization of gaseous media with a conversion efficiency as high as $0.12 \%$ [13-17], even $0.5 \%$ in the case of two-color filamentation [18]. In the filamentation regime, it was suggested that the nonlinear Kerr effect and the reduction of air refractive index due to the plasma may lead to improved phasematching or even phase-locking between the fundamental and third harmonic, resulting in efficient TH generation $[15,19,20]$.

From the point view of its applications for UV pulse generation, optimal experimental conditions for high conversion efficiency are desired. H. Yang and co-workers observed an optimized focal length of $400 \mathrm{~mm}$ for an incident femtosecond laser pulse of $28 \mathrm{~mJ}$ [15]. Unfortunately, in this work the laser beam diameter is not mentioned and hence the focusing geometry is unknown. A. Ionin et al. performed systematic measurement concerning the role of focusing conditions on $\mathrm{TH}$ generation [21]. They reported that the $\mathrm{TH}$ yield increases monotonously with the increase of numerical aperture ranging from 0.004 to 0.06 , without indication as to optimum focusing geometry.

Compared to one single free propagating filament, perturbation of the filament by another control pulses or objects such as waterdrop or glass wedge was found to be able to enhance the TH [22-27]. One intensively studied technique is to use a second femtosecond pulse to intercept the TH generating filament, which leads to a significant enhancement factor ranging from 20 to 700 compared to the case of single pulse filamentation [23, 24]. Other forms of perturbations, including insertion of water droplet or copper fiber in the filament [26], termination of the filament with fused silica wedge or gas pressure gradient [26], also result in $\mathrm{TH}$ enhancement. Concerning the underlying mechanism for these above TH enhancements, different mechanisms have been proposed, such as enhanced third order nonlinear susceptiility $\chi^{(3)}$ or extension of the filament length $[22,23]$. Later, it was suggested that the enhancement arises from the suppression of the destructive interference of the TH generated before and after the focus, preventing the backflow of the TH energy to the fundamental pulse $[25,27]$. In all these experiments, the "enhancement" was defined as the TH yield compared to that obtained with free-propagating filaments and the factor of "enhancement" also depended on the geometrical focusing condition [27]. However, to the best of our knowledge, the absolute energy of the "enhanced" third harmonic was not measured and the improved efficiency of energy conversion is largely unknown. At the same time, it is not clear whether this "enhancement" exists for any focusing conditions.

In this work, we investigated the third harmonic generation in the parameter space of focusing geometry, gas pressure, as well as incident pulse energy, aiming to identify the optimum conditions for TH generation. The existence of an optimal focusing geometry (NA = $0.07)$ for a wide range of incident laser power $\left(0.75 P_{c r} \sim 13 P_{c r}\right)$ was first observed. Furthermore, for different focusing geometries, varying the air pressure leads to distinct behaviors of the $\mathrm{TH}$, pointing out a joint contribution of focusing geometry and gas dispersion for determining the TH conversion efficiency. For the optimal focusing condition, it was found that the $\mathrm{TH}$ generated along the filament accumulates and exits at its end without significant energy backflow to the fundamental pulse. This behavior was confirmed by crossed filaments experiment under different focusing geometries, where NO TH "enhancement" could be observed for the optimal focusing condition.

\section{Experimental setup}

In our experiments, a femtosecond laser system based on chirped pulse amplification was employed. It delivers $800 \mathrm{~nm}, 45 \mathrm{fs}$ pulses with maximum pulse energy of $15 \mathrm{~mJ}$ at a repetition rate of $100 \mathrm{~Hz}$. At the exit of the laser, the laser beam exhibits a waist $\left(1 / \mathrm{e}^{2}\right)$ of 14 $\mathrm{mm}$. The laser pulses are focused by convex lenses with different focal lengths into a $55 \mathrm{~cm}$ long gas chamber, through a $2 \mathrm{~mm}$ thick fused silica window. The fundamental pulse and the 
generated TH exit the gas chamber through a second fused silica window. To measure the $\mathrm{TH}$ pulse energy, the TH pulse centered at $266 \mathrm{~nm}$ is first separated from the fundamental $800 \mathrm{~nm}$ pulse by a fused silica prism. Then, the $\mathrm{TH}$ radiation is focused by an $\mathrm{Al}$ coated parabolic mirror $(f=60 \mathrm{~mm})$ and detected by a calibrated photodiode or a powermeter (Gentec, XLP123S-H2-D0) which is capable of measuring laser power at $266 \mathrm{~nm}$ as low as $5 \mu \mathrm{W}$. To capture the spatial profile of the TH radiation, we insert 2 pieces of short-wave pass glass filters (UG11) into the beam after the exiting windows to filter out the white-light generated after filament while leaving the TH pass through. The TH radiation is then projected on a white paper screen. The fluorescence image of the TH radiation is taken with a CCD camera, which reflects the spatial profile of the TH radiation [27].

\section{Experimental results and discussion}

We first measured the TH energy generated with different focal lengths for laser pulses with energy ranging from $0.15 \mathrm{~mJ}$ to $2.6 \mathrm{~mJ}$, corresponding to the laser power from $0.75 P_{c r}$ to 13 $P_{c r}$. Convex lenses with focal lengths between $100 \mathrm{~mm}$ and $1000 \mathrm{~mm}$ were employed in our study, which correspond to numerical apertures of $0.14-0.014$. The experimental results are presented in Fig. 1. It is seen that the most energetic TH radiation is generated with lenses of $f$ $=200$ for nearly all the pulse energies, with the numerical aperture of 0.07 . For higher incident energy above $1.95 \mathrm{~mJ}$, focusing of $f=300 \mathrm{~mm}$ produces slightly more TH. This observation confirms the existence of an optimal focusing geometry condition reported by Yang et al. [15]. We noticed that the conversion efficiency from $800 \mathrm{~nm}$ to $266 \mathrm{~nm}$ stays rather constant for different incident pulse energies. For the optimal focusing geometry of $f=$ $200 \mathrm{~mm}$, the conversion efficiency is around $0.1 \%$.

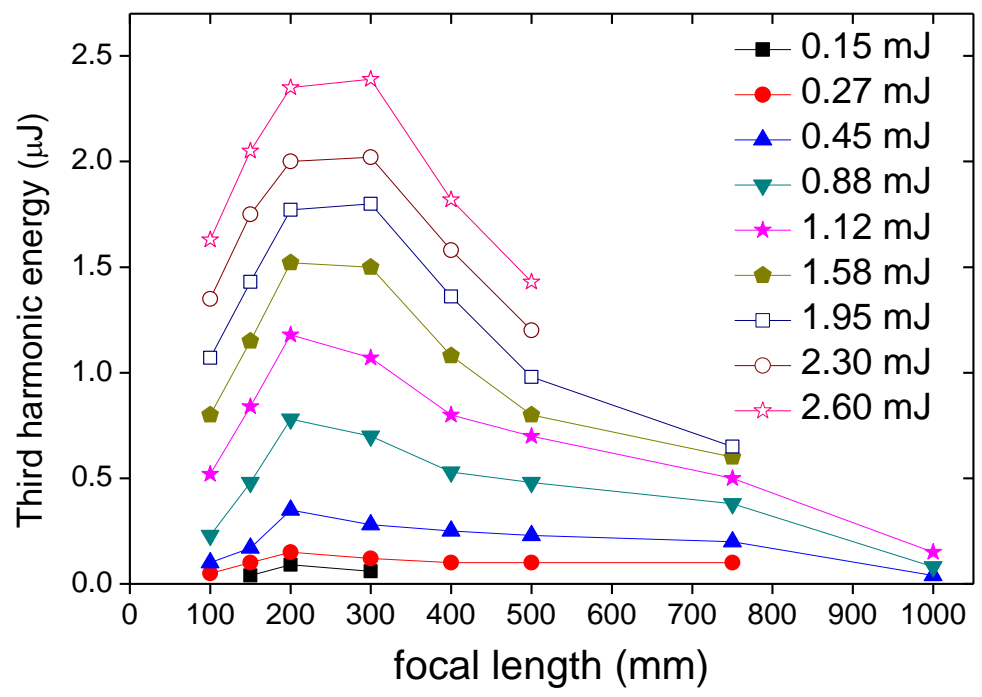

Fig. 1. Third harmonic yield as a function of focusing length for different incident pulse energies. The diameter of the incident pulse is $14 \mathrm{~mm}$.

We noticed that the external focusing geometry has a strong effect on plasma density and can lead to change from linear to nonlinear focusing regime during filamentation in air [28, 29]. To get insight into the existence of the optimal focusing condition, we examined the $\mathrm{TH}$ development along the plasma filament with a scanning pinhole method [19, 27]. We first fabricated a filament-induced pinhole on a $250 \mu \mathrm{m}$ thick aluminum foil by inserting it around the middle of an intense filament. The diameter of the pinhole was found to be about $110 \mu \mathrm{m}$. This pinhole blocks the energy reservoir of filament and thus prevents the further formation of filament after it $[19,27]$. At the same time, the TH generated before the pinhole largely 
passes through since it is mostly generated inside the intense filament core. In the experiments, we scan the position of the pinhole along the filament and monitor the TH yield.

In Fig. 2, the results for focal lens of $f=200 \mathrm{~mm}$ and $1000 \mathrm{~mm}$ are presented. The incident pulses energy was $1.12 \mathrm{~mJ}$ in this experiment. The red arrows in the two panels indicate the $\mathrm{TH}$ yield from free propagating filaments without the pinhole. In the case of the optimal focusing $f=200 \mathrm{~mm}$, the luminous plasma channel is observed to be about $4 \mathrm{~mm}$ long (located from $z=-3 \mathrm{~mm}$ to $1 \mathrm{~mm}$ ) by capturing the image of the plasma fluorescence with a CCD camera [27]. It is found that the TH increases monotonously along the filament from $z=-4 \mathrm{~mm}$ to $z=1 \mathrm{~mm}$. The slight decrease of the TH signal after $z=1 \mathrm{~mm}$ can be due to the partial blockage of the divergent $\mathrm{TH}$ pulse by the $110 \mu \mathrm{m}$ pinhole. The maximum $\mathrm{TH}$ signal observed around the end of the filament is close to that without the pinhole. Therefore, it is obvious that the $\mathrm{TH}$ accumulates along the filament and exits from the end of filament without significant backflow of its energy to the fundamental pulse.
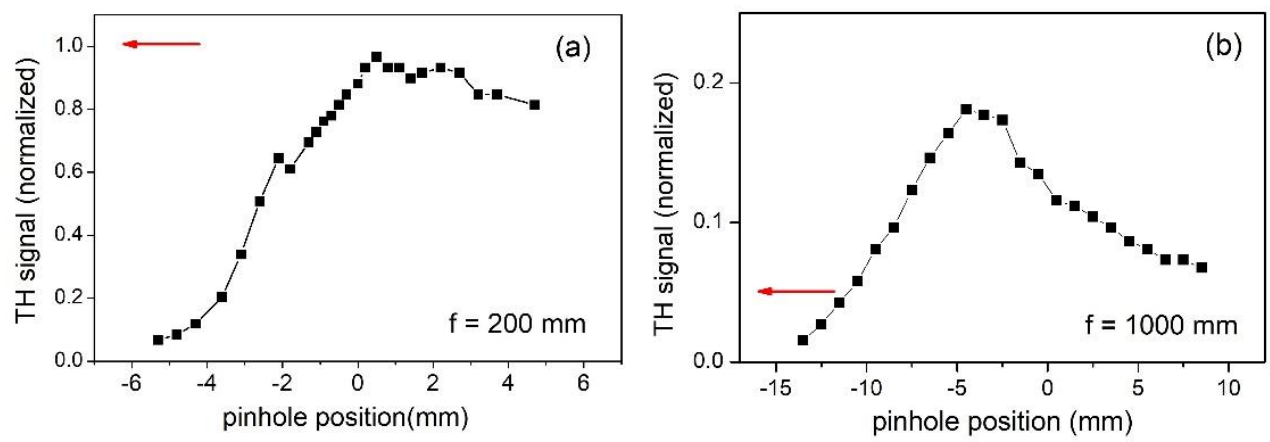

Fig. 2. The third harmonic signal pass through a $110 \mu \mathrm{m}$ pinhole scanned along the filament. (a) $f=200 \mathrm{~mm}$. (b) $f=1000 \mathrm{~mm}$. The origin $(\mathrm{z}=0 \mathrm{~mm})$ is defined as the geometrical focus in each cases.

For $f=1000 \mathrm{~mm}$, the plasma filament is observed about $14 \mathrm{~mm}$ (located from $z=-11 \mathrm{~mm}$ to $2 \mathrm{~mm}$ ). In this case, the development of $\mathrm{TH}$ along the filament is different from the above case. The TH energy first exhibits an increase from $\mathrm{z}=-15 \mathrm{~mm}$ to $\mathrm{z}=-4 \mathrm{~mm}$, with its maximum value $\sim 2.7$ times higher than that from a free propagating filaments without the pinhole. For pinhole positioned in the downstream of the filament, a substantial decrease starting from $\mathrm{z}=-4 \mathrm{~mm}$ is observed. These observations suggest that in the loose focusing condition of $f=1000 \mathrm{~mm}$ the TH is first generated and then gives back its energy to the fundamental pulse [27]. Previous numerical simulation revealed that in the loose focusing conditions the TH energy can oscillate along the long filament, due to the joint action of Gouy phase shift and pressure-dependent wave-vector mismatching [27]. We therefore study the role of air pressure for different focusing conditions.

In Fig. 3, we present the TH yield as a function of air pressure for four different focal lenses. In each case, two incident laser energies are studied. The small irregularity of the TH signal around 250-300 mbar is due to a transition regime between two vacuum gauges used in the experiments. For two different incident pulse energies, similar dependence is observed for the four different focusing conditions.

For the optimal focusing $f=200 \mathrm{~mm}$, reduction of the air pressure results in monotonous decrease of the TH radiation. In contrast, for looser focusing $(f=500,750$, and $1000 \mathrm{~mm})$ a substantial improvement of the TH energy is observed for lower pressures. The optimal pressure decreases systemically with the increase of focal length. In particular, for $f=1000$ $\mathrm{mm}$ the maximum TH energy obtained around air pressure $p=200$ mbar can be $\sim 7$ times higher than that in atmospheric air. For this case of low gas pressure, the pulse propagation can be no longer in the filamentation regime, since the laser peak power now becomes less 
than the pressure-dependent critical power for self-focusing. During this experiment, we also noticed that the spatial profile of the TH emission can be improved for lower gas pressure. The profile
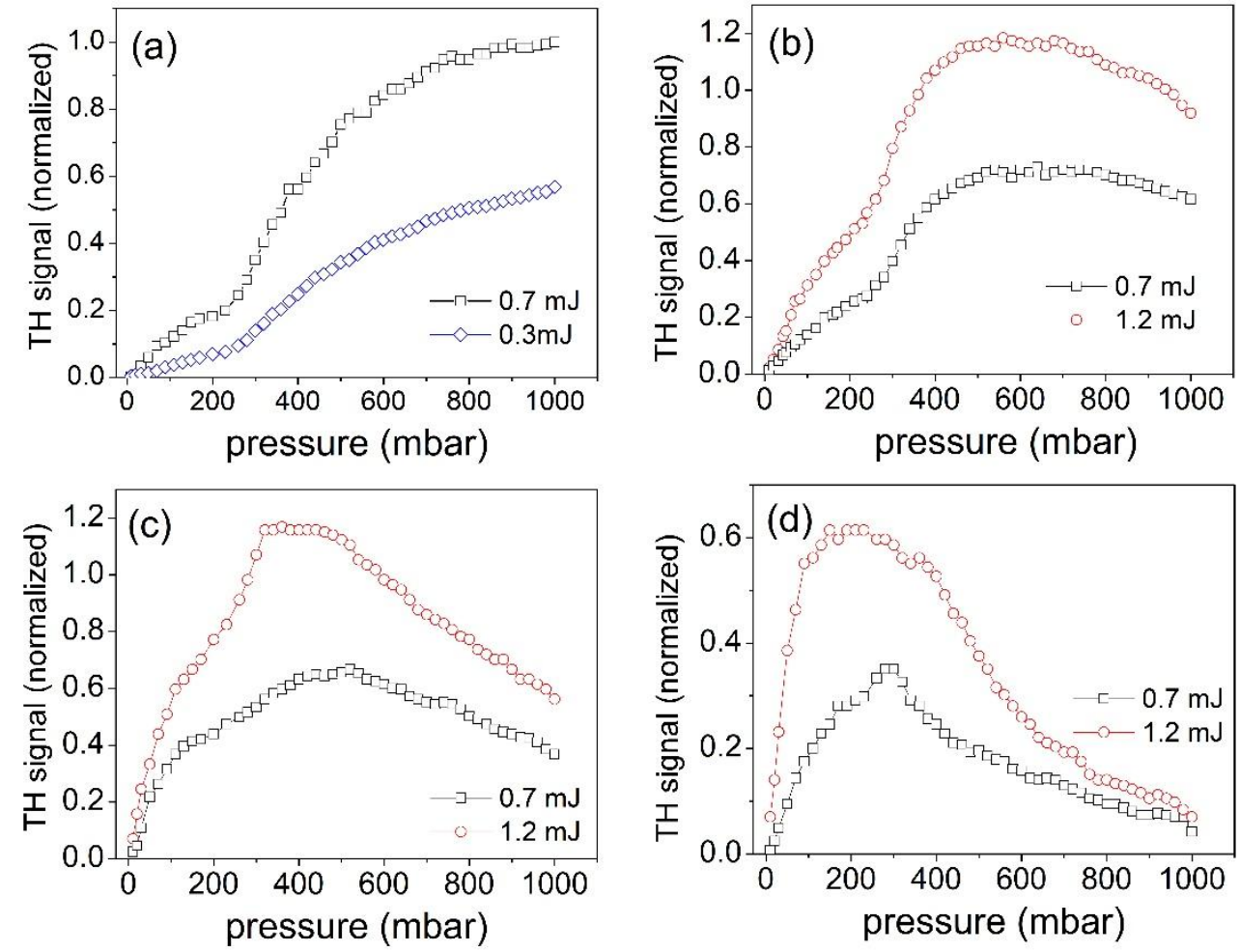

Fig. 3. Third harmonic signal as a function of air pressure for different focal lengths. From (a) to (d), the focal lengths are $200 \mathrm{~mm}, 500 \mathrm{~mm}, 750 \mathrm{~mm}$, and $1000 \mathrm{~mm}$ respectively. All the data are normalized to the signal obtained with $0.7 \mathrm{~mJ}$ incident pulse energy in the case of $f=$ $200 \mathrm{~mm}$ and 1 bar air.

of the TH emission in the case of $f=1000 \mathrm{~mm}$ are shown in Fig. 4. For higher air pressures $(p$ $\geq 300 \mathrm{mbar}$ ), a conical emission with minimum on-axis radiation intensity was observed, which has been widely reported in the filamentation regime [20]. For lower pressures of less than 200 mbar, a much more uniform TH beam profile was observed (Fig. 4 (a)-(c)), which can be beneficial for application.
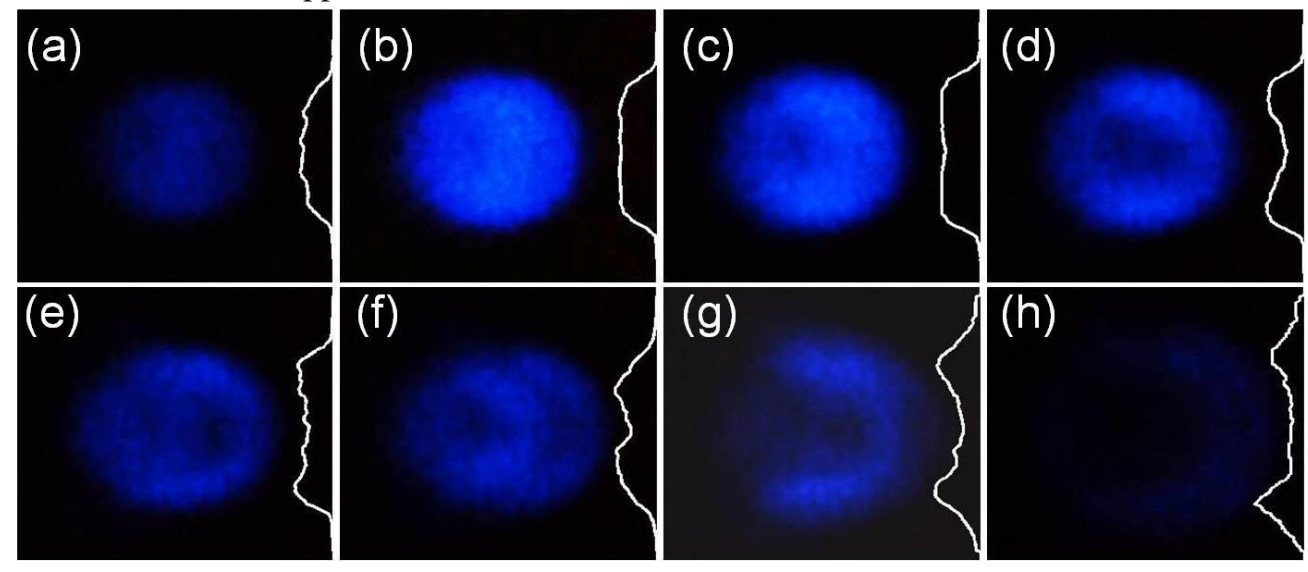
Fig. 4. Far field profile of the third harmonic radiation for different air pressures. The focal length is $1000 \mathrm{~mm}$ and the paper screen is $75 \mathrm{~cm}$ away from the geometrical focus. The incident pulse energy is $1.2 \mathrm{~mJ}$. From (a) to (h), the pressures are 30, 70, 200, 300, 350, 430, 600, 1000 mbar, respectively.

Finally, we applied the crossing filaments method to examine if we can further improve the TH conversion efficiency. In this experiment, the setup was similar to our previous work [27]. The focal lens for the control beam was $500 \mathrm{~mm}$ and the crossing angle between the control beam and the main filamentation beam was about $40^{\circ}$. In Fig. 5, we present the results for two focusing geometries of $f=1000$ and $200 \mathrm{~mm}$. For the loose focusing of $f=$ $1000 \mathrm{~mm}$, a significant enhancement in the spectral domain can be observed with a fiber coupled spectrometer. In this case, the plasma filament of the control pulse provides a refractive index perturbation to the main filamentary pulse and prevents partially the destructive interference effect due to the Gouy phase [27]. In contrast, the situation for the optimal focusing condition $f=200 \mathrm{~mm}$ is different. In this case, no enhancement of the third harmonic energy was observed. This is now understandable since nearly all the TH generated along the filament already exits from the end of filamentary plasma, and hence the plasma perturbation induced by the control filament cannot provide further enhancement.
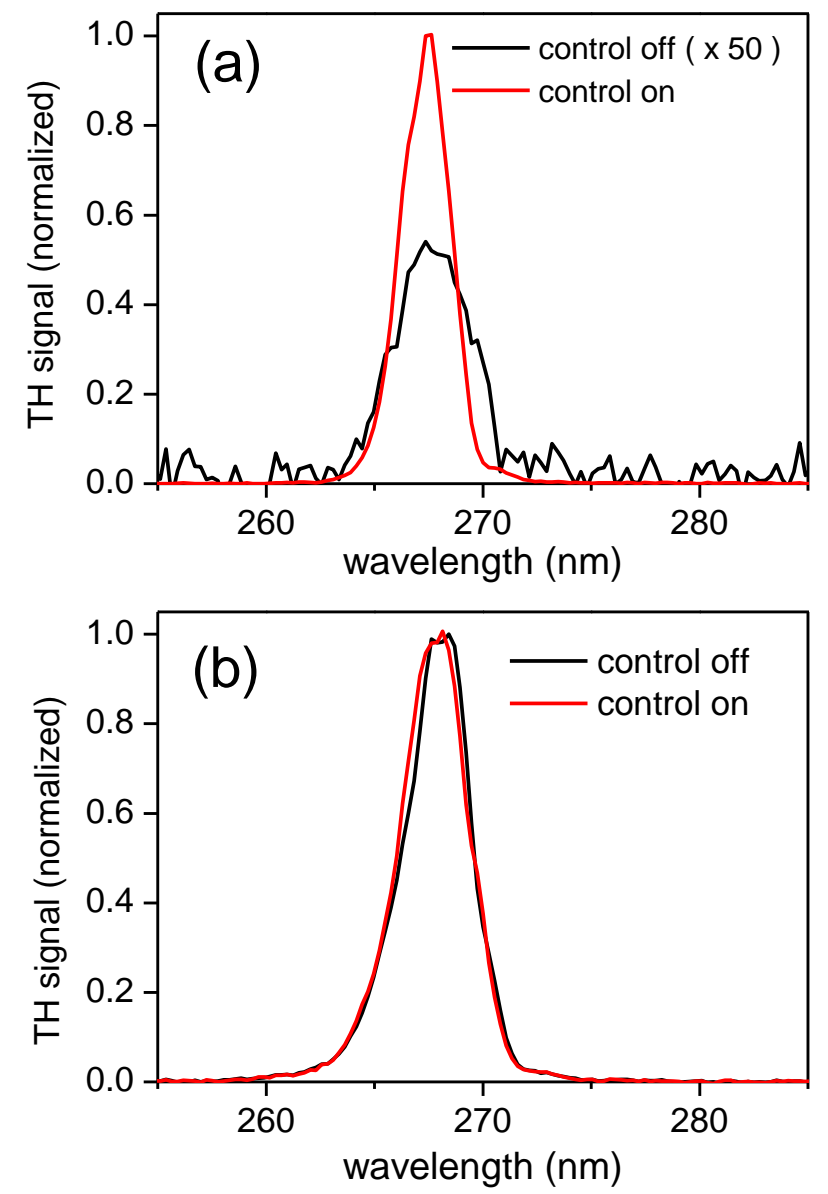

Fig. 5. Effect of crossing filament on the TH generation. The focal lens for (a) and (b) are 1000 $\mathrm{mm}$ and $200 \mathrm{~mm}$ respectively. The focal length for the control pulse was $500 \mathrm{~mm}$ and the control pulse energy was $380 \mu \mathrm{J}$, similar to that in ref [24].

\section{Conclusion}


We have measured the third harmonics yield from air plasma filaments pumped by femtosecond laser pulses as a function of focusing geometry, air pressure, and incident laser pulse energy. For laser peak powers of 0.75 to $13 \mathrm{Pcr}$, an optimal focusing condition of NA = 0.07 was confirmed. With the scanning pinhole method, it was revealed that in the case of optimal focusing, the third harmonic accumulates constructively along the filaments before exiting from the end of filaments, which was also confirmed by the crossing-filaments method. For loose focusing geometry, decreasing the air pressure gives rise to improvement of both radiation energy and beam profile simultaneously.

\section{Acknowledgement}

The work is supported by the National Natural Science Foundation of China (Grants No. 11574213), Innovation Program of Shanghai Municipal Education Commission (Grant No. 2017-01-07-00-07-E00007). Y. Liu acknowledges the support by The Program for Professor of Special Appointment (Eastern Scholar) at Shanghai Institutions of Higher Learning (No. TP2014046) and Shanghai Municipal Science and Technology Commission (No. 17060502500).

\section{References}

1. J. F. Ward and G. H. C. New, "Optical third harmonic generation in gases by a focused laser beam," Phys. Rev. 185(1), 57-72 (1969).

2. G. C. Bjorklund, "Effects of focusing on third-order nonlinear processes in isotropic media," IEEE J. Quantum Electron QE-11(6), 287-296 (1975).

3. R. B. Miles and S. E. Harris, "Optical third-harmonic generation in alkali metal vapors," IEEE J. Quantum Electron QE-9(4), 470-484 (1973).

4. $\quad$ E. R. Peck and K. Reeder, "Dispersion of air," J. Opt. Soc. Am. 62(8), 958-962 (1972).

5. A. Couairon and A. Mysyrowicz, "Femtosecond filamentation in transparent media," Phys. Rep. 441, 47-189 (2007).

6. S. L. Chin, S. A. Hosseini, W. Liu, Q. Luo, F. Theberge, N. Akozbek, A. Becker, V. P. Kandidov, O. G. Kosareva, and H. Schroeder, "The propagation of powerful femtosecond laser pulses in optical media: physics, applications, and new challenges," Canadian Journal of Physics 83, 863-905 (2005).

7. F. Théberge, Q. Luo, W. Liu, S. A. Hosseini, M. Sharifi, and S. L. Chin, "Long-range third-harmonic generation in air using ultrashort intense laser pulses," Appl. Phys. Lett. 87, 081108 (2005).

8. R.W. Boyd, "Nonlinear Optics ( $3^{\text {rd }}$ ed)," Academic Press, San Diego (2008).

9. D. S. Steingrube, T. Vockerodt, E. Schulz, U. Morgner, and M. Kovačev, "Phase matching of high-order harmonics in a semi-infinite gas cell," Phys. Rev. A 80, 043819 (2009).

10. R. Eramo and M. Matera, "Third-harmonic generation in positively dispersive gases with a novel cell," Appl. Opt. 33(9), 1691-1696 (1994).

11. D. S. Bethune and C. T. Retter, "Optical harmonic generation in nonuniform gaseous media with application to frequency tripling in free-jet expansions," IEEE J. Quantum Electron QE-23(8), 1348-1360 (1987).

12. T. Tamaki, K. Midirika, and M. Obara, "Phase-matched third-harmonic generation by nonlinear phase shift in a hollow fiber," Appl. Phys. B 67(1), 59-63 (1998).

13. C. W. Siders, N. C. Turner, M. C. Downer, A. Babine, A. Stepanov, and A. M. Sergeev, "Blue-shifted third harmonic generation and correlated self-guiding during ultrafast barrier suppression ionization of sub atmospheric density noble gases," J. Opt. Soc. Am. B 13(2), 330-336 (1996).

14. S. Backus, J. Peatross, Z. Zeek, A. Rundquist, G. Taft, M. M. Murnane, and H. C Kapteyn, "16-fs, 1- $\mu$ J ultraviolet pulses generated by third-harmonic conversion in air," Opt. Lett. 21(9), 665-667 (1996).

15. H. Yang, J. Zhang, J. Zhang, L. Z. Zhao, Y. J. Li, H. Teng, Y. T. Li, Z. H. Wang, Z. L. Chen, Z. Y. Wei, J. X. Ma, W. Yu, and Z. M. Sheng, "Third-order harmonic generation by self-guided femtosecond pilses in air," Phys. Rev. E 67, 015401 (2003).

16. R. A. Ganeev, M. Suzuki, M. Baba, H. Kuroda, and I. A. Kulagin, "Third-harmonic generation in air by use of femtosecond radiation in tight-focusing conditions," Appl. Opt. 45, 748(2005).

17. A. K. Dharmadhikari, S. Edward, J. A Dharmadhikari, and D. Mathur,"On the generation of polarizationdependent supercontinuum and third harmonic in air," J. Phys. B, 48, 094012(2015).

18. L. Bergé, S. Skupin, G. Méjean, J. Kasparian, J. Yu, S. Frey, E. Salmon, and J. P. Wolf, "Supercontinuum emission and enhanced self-guiding of infrared femtosecond filaments sustained by third-harmonic generation in air," Phys. Rev. E 71, 016602(2005).

19. N. Aközbek, A. Iwasaki, A. Becker, and M. Scalora, S. L. Chin, C. M. Bowden, "Third-harmonic generation and self-channeling in air using high-power femtosecond laser pulses," Phys. Rev. Lett. 89(14), 143901 (2002).

20. F. Theberge, N. Akozbek, W. Liu, J. F. Gravel, and S. L. Chin, "Third harmonic beam profile generated in atmospheric air using femtosecond laser pulses," Opt. Commun. 245, 399-405 (2005). 
21. A. A. Ionin, S. I. Kudryashov, L. V. Seleznev, D. V. Sinitsyn, E. S. Sunchugasheva, and V. Yu. Fedorov, "Third harmonic generation by ultrashort laser pulses tightly focused in air," Las. Phys. 21(3), 500-504 (2011).

22. S. Suntsov, D. Abdollahpour, D. G. Papazoglou, and S. Tzortzakis, "Efficient third-harmonic generation through tailored IR femtosecond laser pulse filamentation in air," Opt. Express 17(5), 3190 (2009).

23. X. Yang, J. Wu, Y. Peng, Y. Tong, S. Yuan, L. Ding, Z. Xu, and H. Zeng, "Noncollinear interaction of femtosecond filaments with enhanced third harmonic generation in air," Appl. Phys. Lett. 95(11), 111103(2009).

24. S. Suntsov, D. Abdollahpour, D. G. Papazoglou, and S. Tzortzakis, "Filamentation-induced third-harmonic generation in air via plasma-enhanced third-order susceptibility," Phys. Rev. A 81(3), 033817 (2010).

25. Z. Zhang, X. Lu, Y. Zhang, M. Zhou, T. Xi, Z. Wang, and J. Zhang, "Enhancement of third-harmonic emission from femtosecond laser filament screened partially by a thin fiber," Opt. Lett. 35(7), 974-976 (2010).

26. J. Yao, B. Zeng, W. Chu, J. Ni, and Y. Cheng, "Enhancement of third harmonic generation in femtosecond laser induced filamentation-comparison of results obtained with plasma and a pair of galss plates," J. Mod. Opt. 59(3), 245-249 (2012).

27. Y. Liu, M. Durand, A. Houard, B. Forestier, A. Couairon, and A. Mysyrowicz, "Efficient generation of third harmonic radiation in air filaments: A revisit," Opt. Commun. 284(19), 4706-4713 (2011).

28. F. Théberge, W. Liu, P. Tr. Simard, A. Becker, and S. L. Chin, "Plasma density inside a femtosecond laser filament in air: Strong dependence on external focusing," Phys. Rev. E 74, 036406 (2006).

29. K. Lim, M. Durand, M. Baudelet, and M. Richardson, "Transition from linear- to nonlinear-focusing regime in filamentation," Sci. Rep. 4, 7217(2014). 